\section{Web Site Development \\ at a Museum Microscopy Laboratory}

David P. Reddy, Angela V. Klaus, Renée Recker, and William

K. Barnett, American Museum of Natural History

The Interdepartmental Laboratories (IDL) is the core microscopy and scientific visualization research facility for the American Museum of Natural History (AMNH). Its purpose is to operate and maintain facilities that have broad application within the Museum and would be either too costly for the individual research departments to maintain or which would be underused in a typical department setting. The IDL currently maintains two state-of-the-art analytical/imaging microscopes, as well as resources for visualization, webaccessible databases, and networked image scanning, archiving, and printing. The IDL is overseen by Bill Barnett, the IDL Director, and staffed by Angela Klaus, the Laboratory Manager, and David Reddy, the Scientific Visualization and Informatics Manager.

With the recent explosive growth of Internet technologies and their permeation throughout society, web sites have become virtually required for individuals, departments, institutions, and companies involved in every facet of human endeavor. We will present the specification and creative design processes of a web site for our microscopy and visualization lab, along with the software used in creating and organizing the site's visual content. IDL undertook this process in conjunction with Renée Recker, a commercial web site designer and volunteer at the Museum.

The IDL web pages (http://research.amnh.org/id/) are designed to serve several constituencies: research scientists at the Museum, the general Museum community including the education and exhibitions departments, researchers and microscopists worldwide, and the general public. IDL's goal in developing the web pages was to provide detailed information about our facilities and research applications. These pages are also designed to expedite the efficient use of our resources and provide equipment specifications for grant writing and publications for scholars, internal or external, using our facilities. At a broader level, we needed to communicate that the lab produces images and visualizations of analyzed specimens both to catalyze new research applications and to inform non-scientific constituencies about the lab's purpose and contributions. To meet these goals, it was important that our web site first focus on descriptions of the equipment and research applications.

\section{Microscopy and Museum Applications}

Biological applications for scanning electron microscope (SEM) imaging range from describing specimen morphologies for taxonomic research, to studying fossil mineralization, and studying growth and developmental morphology. Our recently acquired Hitachi S-4700 Cold Field Emission SEM (NSF grant \# DBI-9724454) enables our researchers to image uncoated, non-conductive, biological specimens. Uncoated, non-destructive sample preparation is essential when viewing "type" specimens. Type specimens are those that taxonomically define a particular species or family, so metal coating cannot be used, as it would alter them. The Museum's collection of type specimens runs the gamut of faunal biodiversity and includes numerous significant species of insects, spiders, vertebrates, invertebrates, and fossils. Many type specimens are of rare species, with only a very few examples known in existence; and some are extinct. Many morphological characteristics of these specimens are describable only by SEM, thus making the instrument indispensable to research at the Museum.

The Hitachi FE-SEM is equipped with a Princeton Gamma Tech energy dispersive spectroscopy (EDS) $x$-ray microanalysis system and a Centaurus backscattered electron detector. This system allows our researchers to perform qualitative and quantitative $x$-ray microanalysis, $x$-ray mapping, line scanning, and image analysis. We plan to add cathodoluminesence capabilities as well.

The Earth and Planetary Sciences Department at the AMNH uses SEM/

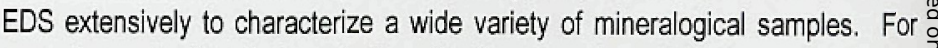
example, meteorites that are believed to have condensed at the same time as $\frac{\overline{5}}{5}$ the solar system are called "chondrules". Determining chondrule chemistry helps researchers understand the conditions that existed in the early solar system. The $\frac{\rho}{3}$ Anthropology Department makes use of the SEMIEDS system to characterize various artifacts housed by the Museum. The morphology of textiles and hair ${ }_{c}^{\circ}$ and the chemical composition of pigments, glasses, and metals are valuable clues when trying to determine the origin and nature of anthropological specimens and determine conservation treatments.

The AMNH's confocal microscopy lab currently houses a Zeiss 510 Confocal Laser Scanning Microscope (CLSM). This instrument's computer control over the lasers, pinholes, and detectors is extremely useful, allowing the user to concentrate more fully on imaging. Museum scientists are using this microscope to study evolution by looking at the expression of certain proteins within developing invertebrates and insects, localized within an organism or cell using immunofluorescence labeling. Other applications include the characterization of fluid inclusions in igneous melts. A less traditional use for confocal microscopes, but one that is gaining popularity, is the imaging of non-transparent surfaces and objects, which relies on the use of a reflected light signal. We are using this technique to do quantitative measurements on small, opaque specimens such as shells, teeth, skulls, and insects.

\section{Scientific Visualization and Image Processing}

The ability to manipulate and analyze $2 \mathrm{D}$ and $3 \mathrm{D}$ data is becoming a critical adjunct to the scientific enterprise at the Museum. The IDL has a Silicon Graphics Onyx@ workstation with IBM Visualization Data Explorer ${ }^{\text {Th }}$ and other visuali-

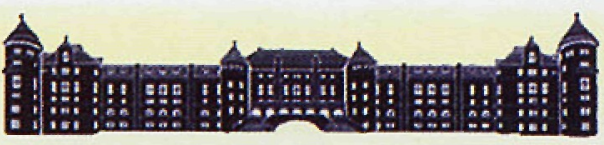

American Museum of Natural History

INTERDEPARTMENTAL LABS

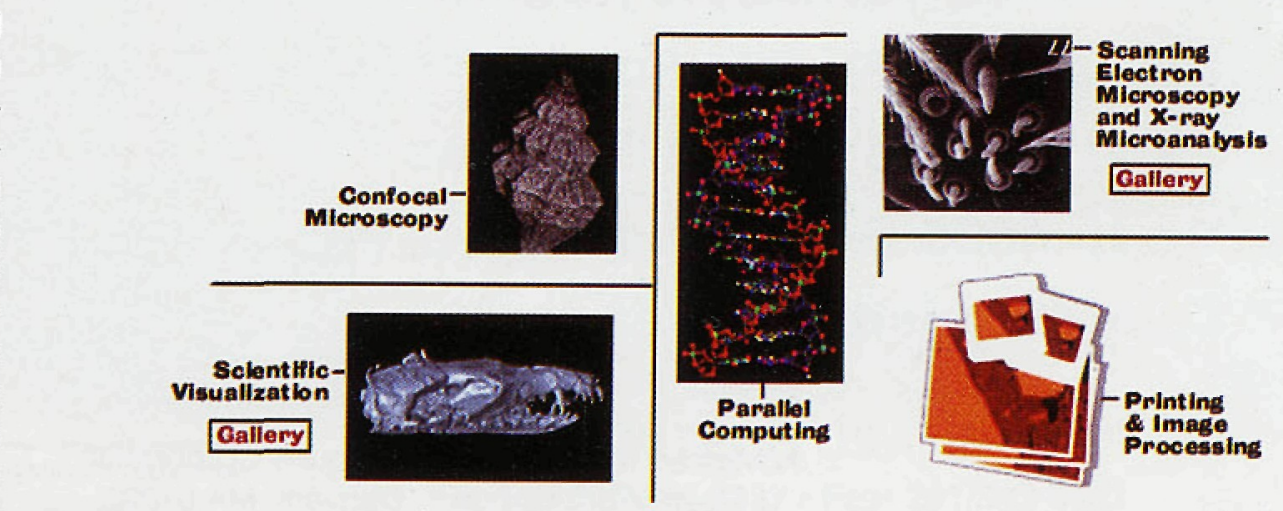


zation software for working with 3D data sets. These sets include image volumes from our confocal microscope, other 3D data sets such as CT scans, and $3 \mathrm{D}$ representations of statistical analyses of morphometric research. These visualizations and data sets are part of an overall informatics effort to provide webaccessible research databases to our scholars and their collaborators worldwide.

The lab also provides resources for manipulating, storing, archiving and printing digital images. These facilities include a scanner, various printers (up to photo resolution), a film recorder, workstations for scientists, and a disk array for image storage and a CD-ROM writer for archiving data. All instruments are run on standard computer platforms linked over the Museum's computer network to a central disk array of $30 \mathrm{~GB}$ for pre-archive storage. These facilities are also accessible from workstations throughout the Museum.

\section{The Web Site Production Process}

Although the IDL already had a web site, it was one which, like many, had been created over time by editing Hypertext Markup Language (HTML, the lingua franca of the World Wide Web) files with a text editor. It was mostly text oriented, with little image content and no cohesive visual style - it was starting to look a bit dated by today's standards. Renée Recker, a visual artist and web site designer, had been volunteering at the AMNH redesigning departmental web sites under the auspices of the IDL. She had become interested in microscopy, particularly in the colorizing of SEM images, and volunteered to participate in the redesign of the IDL web site.

The process of specifying the content and structure of the new IDL site began with a meeting between Renée and the IDL staff. We first discussed the goals of the site, how other staff members and we felt about the existing site's shortcomings, the constituencies it should serve, and how our new web site might better serve them. We drew a chart of the organization of the labs, which helped determine the site structure, the internal pages and their headings, and also the navigation bar structure. The IDL staff then wrote text, took digital photographs of the equipment, and produced microscopy images and computer renderings for illustrating the web pages.

The challenge facing Renée, as the designer, was how to communicate scientific and technical information in an efficient and appealing style. Typically, the front or "splash" page is graphically the strongest page in a web site, the page where the visual organization starts and the stylistic tone is set for lower-level "internal" pages. It must also present a coherent interface that is not too image-laden, and that can be followed logically and in an easily navigable fashion throughout the site. At the first meeting, we decided to use digital photos of the equipment, such as the microscopes, to illustrate the capabilities of the IDL. Unfortunately, this produced a design that seemed cold and lacked interest and we concluded that a redesign was necessary. At a second meeting, we decided to use images of specimens for the visual content.

The outcome of the redesign was a clean, white, modernistic front page better suited to the mission and spirit of the IDL (See Figure 1: The IDL Home Page [homepage.tiff]). A simplified graphic of the 77th Street façade of the Museum provided by the Graphics Department, with "American Museum of Natural History" in the Museum's official font, DeVinne, serving as the heading, and "INTERDEPARTMENTAL LABS" in a block of color below it. Underneath this, the biological images representative of the various IDL facilities were used as clickable icons to guide users to the internal pages. Additional graphic design elements were simple blocks of color and thin lines. At the bottom, a few text links were used for linking to pages whose functions were not easily illustrated by the use of imagery, such as the staff listing, and to other areas of the Museum. This design was accepted with minor revisions.

Continued on following page
American Museum of Natural History INTERDEPARTMENTAL LABS scanning electron microscopy and $\mathrm{x}$-ray microanalysis

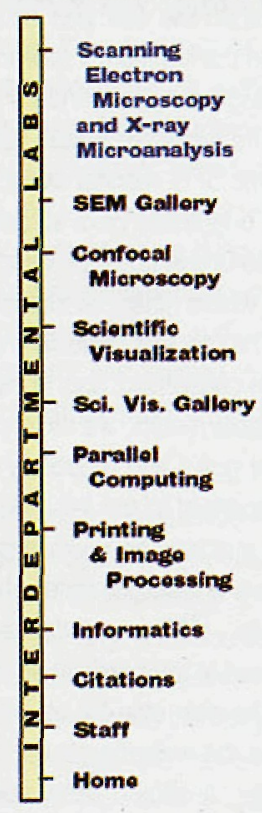

\section{Scanning Electron Microscopy and X-ray Microanalysis at the American Museum of Natural History}

\section{Scanning Electron Microscopy Laboratory: Instrumentation}

\begin{abstract}
The Museum has recently acquired a Hitachi $\$ 4700$ Field Emission Scanning Electron Microscope (FE-SEM). An outstanding feature of this state-of-the-art instrument is that it enables our researchers to image non-conductive specimens without first having to deposit a conductive coating on the sample. Such non-destructive imaging is essential when viewing "type" specimens. The FE-SEM is equipped with a PGT $x$-ray microanalysis system. This system allows our researchers to perform qualitative and quantitative $x$-ray microanalysis, $x$-ray mapping, line scanning, and image analysis. A unique feature of our combination of instrumentation is the ability to do high spatial resolution x-ray microanalysis. Backscattered electron imaging is also possible on the FE-SEM using the GW Centaurus BSE detector. The backscattered electron signal yields contrast based on differences in average atomic number.
\end{abstract}

\section{Overview of Scanning Electron Microscopy}

The Museum's Hitachi 4700 Field Emission Scanning Electron Microscope (FE-SEM) enables researchers to investigate specimens in detail that far exceeds that of light microscopy. The SEM, which scans an electron beam across the surface of a specimen, yields magnifications that can easily exceed $100,000 \mathrm{x}$, far beyond the $1,000 \mathrm{x}$ maximum magnification of light microscopy. Moreover, depth of field is many times greater than that possible with light microscopy. These unique qualities allow images of startling clarity and definition, making the SEM a perfect 


\section{Web Site Development}

Continued from preceding page

For the internal pages, Renée created artwork for each page heading in the same clean modern approach as the front page, using lines and blocks of color as design elements (See Figure 2: The Scanning Electron Microscope and X-Ray Microanalysis second level page [homepage.tiff]). Intuitive and quick navigation between a related set of web pages speeds understanding and enjoyment of a web site. The IDL staff decided they wanted a "navigation bar" on the left side of each internal page to allow users to get to any other internal page without returning to the front page. The artwork for the navigation bar was simple enough to carry the long titles of the various IDL functions. A sample page was laid out using a heading, navigation bar, text and pictures. Upon approval, this page became the standard layout for all the other pages

Each page was then created following the original layout with individual elements as appropriate. Instead of using the text from the old web pages, the IDL staff provided revised or completely rewritten text. The digital camera photos were used to illustrate the microscopes and other equipment. Gallery pages were added to give users access to sample SEM images and computer visualizations of confocal and other $3 \mathrm{D}$ data sources, which the IDL staff could easily maintain and update.

\section{Software Used in the Production Process}

A number of software programs were used in the creation of this web site. The photographic elements and type on the front page were "layered" in Adobe Photoshop 5.0. Layering is a technique whereby individual graphic elements can be moved and edited individually and then composited into a final graphic, as if they were each on a transparent sheet. The layers were composited or "flattened" and converted to the GIF format to be web-ready.

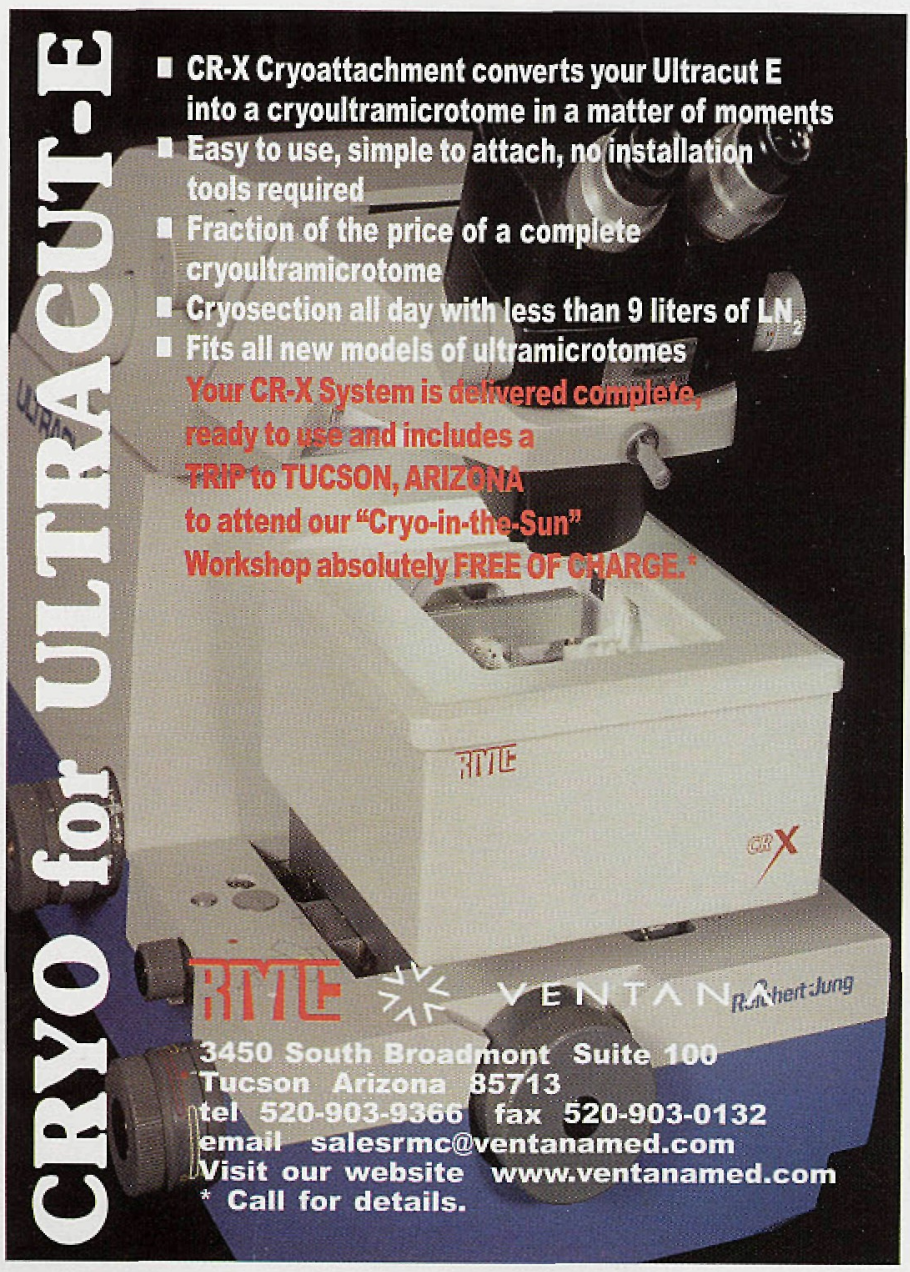

Photoshop was also used to convert many images to JPEG for the internal pages. The headings and navigation bars were made within Adobe Illustrator 7.0, where clean lines and blocks of color were easier to create than in Photoshop. The artwork was made ready for the web by exporting them to GIF format.

GIF format is used in web documents where precise rendering or graphic elements is more important than raw speed of transfer, because there is no loss of image quality in the format. The JPEG format uses a variable "lossy" com-o pression which can greatly reduce image storage size, at the expense of spatiali and color resolution, making it more useful for pictures with large numbers of

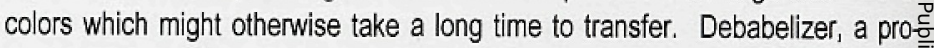
gram which can further reduce image size and rendering time by eliminating seldom used colors and optimizing image color maps, was used to reduce theo size of photographs that were downloading too slowly.

The actual page building process was done in Macromedia Dreamweaver 1.2. The web-ready, formatted photographs, artwork and text were inserted intos a standard page layout or "table". These tables held all the elements together inc a cohesive design, which was output in HTML format when complete.

Fetch, a freeware file transfer protocol (FTP) program for the Apple Macintosh (Dartmouth University) was used to download all the art elements used in₹ the page layouts, and to upload them to Renée's web site for evaluation. A simple FTP program is incorporated into Windows 95 and later versions for PC users. When the site was complete and approved, it was uploaded to become "live" on the Museum's research web server, a Sun Sparc Ultra-1 running Solaris 2.6, using the Apache Version 1.2.6 web server, where it currently resides.

The hardware Renée used was a Macintosh G3 with 160 MB RAM, a NEC Multisync 5FG monitor set to PC gamma (the relation between input and displayed brightness on a screen), and a 256 color display to reflect the low color resolutions that could be used to view the site. All design software mentioned is, except where noted, available for both PC and Macintosh.

The process of designing and creating a web site for a microscopy lab can be time-consuming and complicated. While there are many ways to achieve acceptable results, we have presented a design method and software tools, that met our goals and (hopefully) resulted in a web site that serves the constituencies important to our lab. We also presented an overview of some of the challenges that may be encountered in designing a microscopy site. Each institution will have a different style, philosophy and environment to try to represent with their site. With a well-designed web site, these unique characteristics can be presented to the public in an attractive and informative format.

The next step for us is to get feedback from our constituencies in order to improve the web site. In the future, we look forward to providing a more interactive site. It should allow scientists to schedule microscope time interactively, provide access to their archived images, and support our plans for remote control or remote viewing of our microscope screens. We also plan to add a bibliography of publications that use results from our lab, and develop educational outreach programs for the lab.

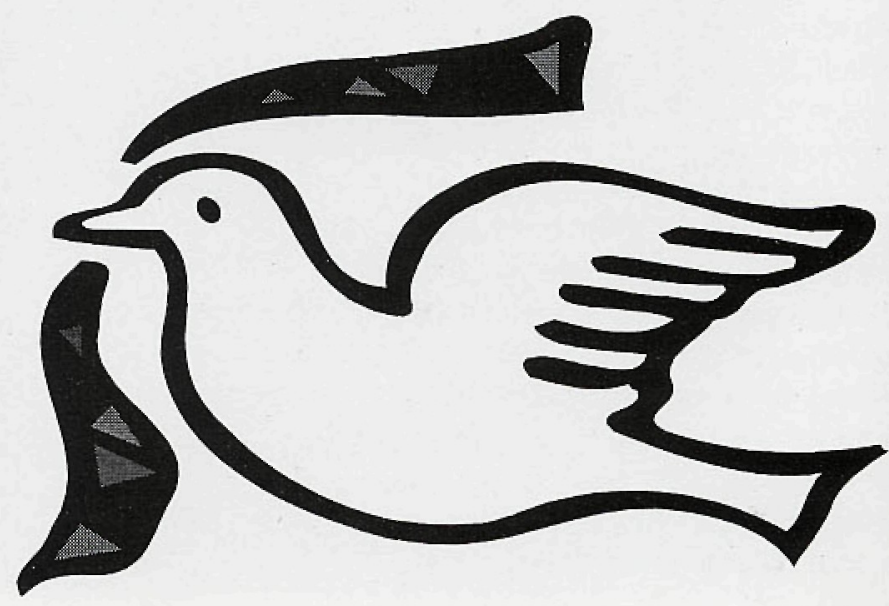




\section{O n e B e a m}

$M$ a $n$ y

\section{O n e Co m}
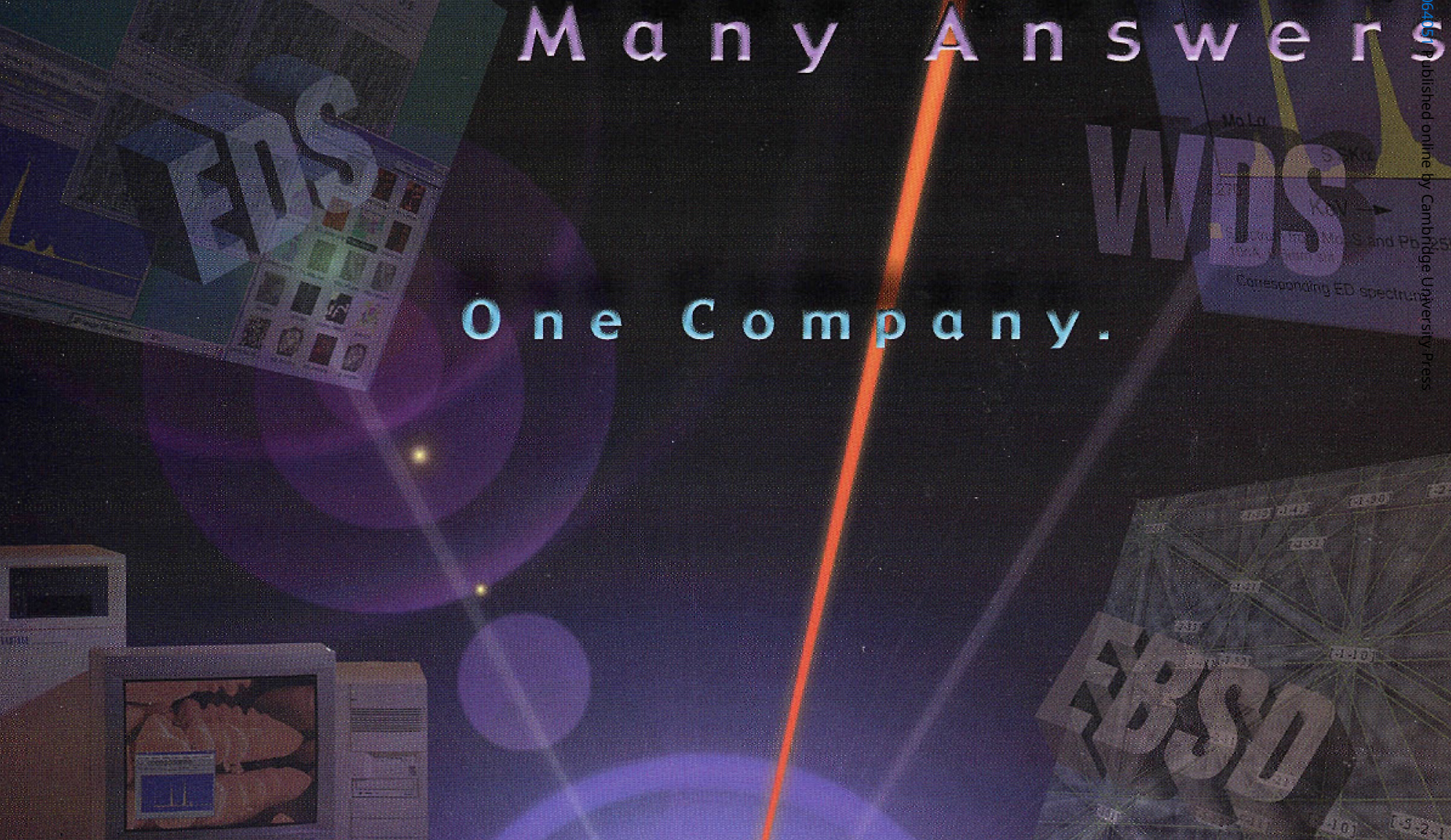

.

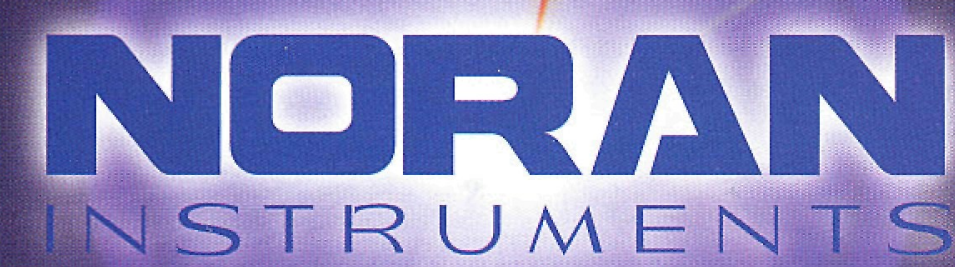

Count On Us

NORAN Instruments Inc., 2551 West Beltline Hwy., Middleton, WI 53562-2697, USA Tel.: 608831 6511, Fax: 6088367224 Internet: micro_info@noran.com, Web: http://www.noran.com

NORAW Instruments B.V., Tel.: 3135 6998888, Fax: 31356949913

WORAN Instruments Gmbl., Tel:: 497251 97910, Fax: 497251979111

NORAN Instruments Japan., Tel.: 81442466190 , Fax: 81442466191

NOBAW Instruments U.K., Tel.: 01869327711 , Fax: 01869327744 